\title{
Cellular Automata Modeling of Microcrystalline Structure Formation
}

\author{
Mikhail Mikolaychuk ${ }^{1, \text { a) }}$ and Anna Knyazeva, 2, b) \\ ${ }^{1}$ Institute of Strength Physics and Materials Science SB RAS, Tomsk, 634055, Russia \\ ${ }^{2}$ National Research Tomsk Polytechnic University, Tomsk, 634050, Russia \\ a) Corresponding author: mihail@mikolaichuk.com \\ b)anna@ispms.tsc.ru
}

\begin{abstract}
The paper discusses the problem of microcrystalline structure formation during magnetron sputtering. The authors suggest the model based on cellular automata approach combined with chemical kinetics considerations, briefly discuss a practical way for kinetic constants evaluation and derive their accurate values. The work numerically solves a particular problem of coating formation by magnetron sputter deposition in multicomponent chemically active plasma.
\end{abstract}

Keywords: cellular automata, simulation, microcrystalline structure, magnetron, plasma

\section{INTRODUCTION}

The problem of microcrystalline structure formation is of great importance in materials science. The finite structure and the process time evolution affect significant mechanical properties of materials. Microcrystalline structure can change during melting, recrystallization, solidification, etc. Such phase transitions take place in a many technical processes like alloy casting, annealing, press forming, coating deposition.

One of the most popular coating methods is the magnetron sputter deposition which allows producing thin coatings from different types of materials [1]. The coatings can be obtained in both inert and reactive atmosphere. In the second case, the reacting gasses can be added to the inert gas (usually argon). From this point of view, any coating is a product of chemical reactions taking place in plasma.

There are many methods that allow simulating the microstructure evolution during phase transitions. Some of them deal with continuous medium, for instance phase field method [2]. Others like Monte-Carlo method [3] or method of cellular automata are based on discrete approach. In this work, we use the modified cellular automata method. Each automata represents the elementary volume filled by a particular chemical compound and the evolution of individual automaton is described by a system of ordinary differential equations of chemical kinetics.

\section{PROBLEM STATEMENT}

We considered the coating formation on a specimen surface by magnetron sputter deposition. We assume the initial nuclei to exist in the considered area. In the neighborhood of the nuclei the formation of the following three crystalline compounds can occur: $\mathrm{TiNi}, \mathrm{Al}_{2} \mathrm{O}_{3}, \mathrm{Si}_{3} \mathrm{~N}_{4}$. Plasma has similar composition in each point of the area $(\mathrm{Ti}$, $\mathrm{Ni}, \mathrm{Al}, \mathrm{O}_{2}, \mathrm{Si}$ ). The following chemical reactions leading to the coating formation were considered:

$$
\mathrm{Ti}+\mathrm{N} \stackrel{\phi_{1}}{\rightarrow} \mathrm{TiN}, \quad 2 \mathrm{Al}+3 \mathrm{O} \stackrel{\phi_{2}}{\rightarrow} \mathrm{Al}_{2} \mathrm{O}_{3}, \quad 3 \mathrm{Si}+4 \mathrm{~N} \stackrel{\phi_{3}}{\rightarrow} \mathrm{Si}_{3} \mathrm{~N}_{4} .
$$

Mass changing rate can be defined as

International Conference on Physical Mesomechanics of Multilevel Systems 2014
AIP Conf. Proc. 1623, 419-422 (2014); doi: 10.1063/1.4898971

(C) 2014 AIP Publishing LLC 978-0-7354-1260-6/\$30.00 


$$
\frac{\mathrm{d}}{\mathrm{d} t} \int_{V} \rho_{i} \mathrm{~d} V=\int_{V} \frac{\partial \rho_{i}}{\partial t} \mathrm{~d} V
$$

where $i$ is a component number $(i=1, \ldots, 8), \rho_{i}$ is a component density. In general, the integral (2) equals to the sum of mass coming through the surface of local volume and the sum of substance produced in a chemical reaction. However, high rate of the sputtering process leads to diffusion evening out between elementary volumes:

$$
\int_{V} \frac{\partial \rho_{i}}{\partial t} \mathrm{~d} V=\sum_{n=1}^{3} \int_{V} \mu_{i} v_{i n} \phi_{n} \mathrm{~d} V .
$$

Here $\mu$ is molar mass of the $i$-th component number, $v_{\text {in }}$ is stoichiometric coefficient of the $i$-th component in the $n$-th reaction (positive for products and negative for reagents), $\varphi_{\mathrm{n}}$ is a rate of the $n$-th chemical reaction.

For the system of reactions (1) the rates can be defined as

$$
\phi_{1}=k_{1} C_{T i} C_{N}, \phi_{2}=k_{2} C_{A l}^{2} C_{O}^{3}, \phi_{3}=k_{3} C_{S i}^{3} C_{N}^{4},
$$

where $C$ is mass concentration of the corresponding system component, $k$ is the constant of chemical reaction rate that generally depends on the environment properties, specimen surface structure and temperature. Mass concentration can be defined as

$$
C_{i}=\frac{\rho_{i}}{\rho} .
$$

Let us derive the mass-balance equation for any component of our system using the total derivative definition:

$$
\frac{\mathrm{d} C_{i}}{\mathrm{~d} t}=\frac{1}{\rho} \sum_{n=1}^{3} \mu_{i} v_{i n} \phi_{n},
$$

where $\rho=C_{i} \rho_{i}$. For reactions like

$$
p A^{i}+q B^{i} \rightarrow A_{p}^{i} B_{q}^{i},
$$

the chemical rate constant can be written according to the theory of chemical kinetics,

$$
k_{i}=e\left[\frac{R T}{N_{\mathrm{A}} h}\right] \exp \left[\frac{\Delta S_{i}^{0}}{R}\right] \exp \left\lfloor-\frac{E_{\mathrm{a} i}}{R T}\right\rfloor,
$$

where $N_{\mathrm{A}}$ is Avogadro's number, $h$ is Planck's constant, $\Delta S_{i}^{0}$ is the reaction entropy $E_{\mathrm{a} i}$ is activation energy,

$$
\begin{gathered}
\Delta S_{i}^{0}=S\left(m A^{i}\right)+S\left(n B^{i}\right)-S\left(A_{m}^{i} B_{n}^{i}\right), \\
E_{\mathrm{a} i}=T_{\mathrm{s}}\left(\Delta S_{i}^{0}+R\right),
\end{gathered}
$$

$R$ is universal gas constant, $T$ is the process temperature or its average value, $T_{\mathrm{s}}$ is standard temperature (298 K). Thereby, to define the required value of the reaction rate constant we need to know the standard entropy of reagents and the temperature of the process. Formation entropy can be defined as [4]

$$
S=R\left(\frac{3}{2} \ln A_{\mathrm{ev}}+\ln V_{\mathrm{ev}}-\frac{3}{2} \ln T_{\mathrm{ml}}\right)+a,
$$

where $A_{\mathrm{ev}}$ is average atomic weight, $V_{\mathrm{ev}}$ is average atomic volume, $T_{\mathrm{ml}}$ is melting temperature, $a$ is a constant that equals to $52.335 \mathrm{~J} /(\mathrm{mol} \cdot \mathrm{K})$. The reaction rate constants were calculated according to the formulas (5)-(8) in the dimensionless form: $k_{1}=1.64, k_{2}=1.00, k_{3}=7.50$. 


\section{MODIFIED CELLULAR AUTOMATA METHOD}

We considered the three-dimensional unit volume of a real sample divided into elementary volumes. This volumes are identical, since each of them comprise plasma of the same composition. At the initial time the nucleation centers were randomly distributed. From our point of view, the nucleation center is the element with nonzero concentration of one of the products at initial moment of time. Assuming that the components can not dissolve in each other, we suggest that at any time thereafter only "nucleation" compound can form in this element. The reagents that do not take part in the formation of this compound will remain unbound [5].

We suppose that, by analogy with the method of cellular automata, the state of the elementary volumes depends on the state of its neighbors. That is, the compound in a single elementary volume shall form depending on the mean concentration of reaction products in adjacent elements. In general, it means that we should analyze the state of 26 adjacent elements for each automaton (excluding boundary elements).

On the other hand, the reaction will proceed more intensively in the vicinity of a larger amount of unreacted reactant. To estimate this quantity, we introduce the parameter $\eta$, the fraction of empty adjacent elements which contain reagents only. The parameter $\eta$ is equal to 1 when all neighbors are not occupied, and to 0 , if there are no empty elements around. Summarize everything above, one can formulate the system of ordinary differential equations according to equations (3) and total derivative definition:

$$
\begin{aligned}
& \frac{\mathrm{d} C_{\mathrm{Ti}}}{\mathrm{d} t}=-w \eta \mu_{\mathrm{Ti}} \phi_{1}, \frac{\mathrm{d} C_{\mathrm{N}}}{\mathrm{d} t}=-w \eta \mu_{\mathrm{N}}\left[\phi_{1}+4 \phi_{3}\right], \frac{\mathrm{d} C_{\mathrm{Al}}}{\mathrm{d} t}=-w \eta \mu_{\mathrm{Al}} 2 \phi_{2}, \frac{\mathrm{d} C_{\mathrm{O}}}{\mathrm{d} t}=-w \eta \mu_{\mathrm{O}} 3 \phi_{2}, \\
& \frac{\mathrm{d} C_{\mathrm{Si}}}{\mathrm{d} t}=-w \eta \mu_{\mathrm{Si}} 3 \phi_{3}, \frac{\mathrm{d} C_{\mathrm{TiN}}}{\mathrm{d} t}=w \eta \mu_{\mathrm{TiN}} \phi_{1}, \frac{\mathrm{d} C_{\mathrm{Al}_{2} \mathrm{O}_{3}}}{\mathrm{~d} t}=w \eta \mu_{\mathrm{Al}_{2} \mathrm{O}_{3}} \phi_{2}, \frac{\mathrm{d} C_{\mathrm{Si}_{3} \mathrm{~N}_{4}}}{\mathrm{~d} t}=w \eta \mu_{\mathrm{Si}_{3} \mathrm{~N}_{4}} \phi_{3},
\end{aligned}
$$

where $w=\rho^{-1}$.

The problem was solved numerically. Each ordinary differential equation was solved using the $4^{\text {th }}$ order RungeKutta method. The solution involved the Odeint numerical library which makes part of Boost library repository.

\section{RESULTS}

Calculations were performed for $N=100$. The process temperature was assumed to be constant and being equal to $T=600 \mathrm{~K}$. The plasma composition was as follows: $10 \%$ of $\mathrm{Ti}, 40 \%$ of $\mathrm{Ni}, 10 \%$ of $\mathrm{Al}, 10 \%$ of $\mathrm{O}_{2}$ and $20 \%$ of $\mathrm{Si}$. Six initial nuclei were randomly located in the considered area (two nuclei for each compound). The calculations were performed until there were no empty elements in the considered area. All calculations were performed in dimensionless form. Numerical realization was tested for approximation convergence and stability. As a result, we have chosen the time step $t_{\mathrm{h}}=0.00001$.

The finite microcrystalline structure shown in the cross sections $x=0.5, y=0.5$ and $z=0.5$ (Figs. $1-3$ ). It is obviously that titanium nitride is dominating. This behavior has two different reasons.

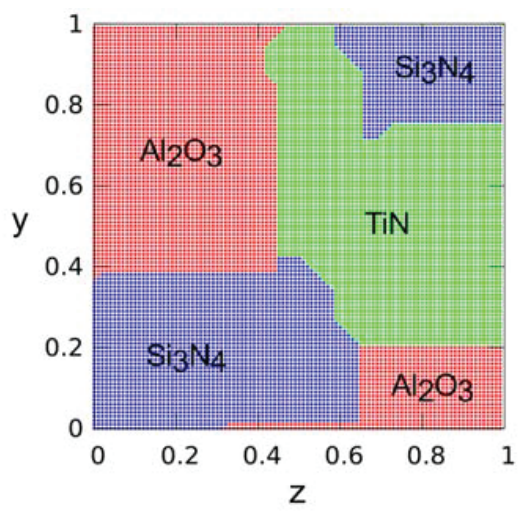

FIGURE 1. Crystalline structure in the cross section $x=0.5$

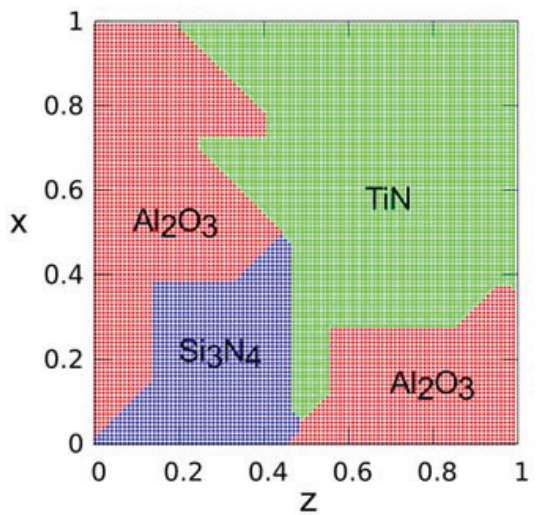

FIGURE 2. Crystalline structure in the cross section $y=0.5$ 


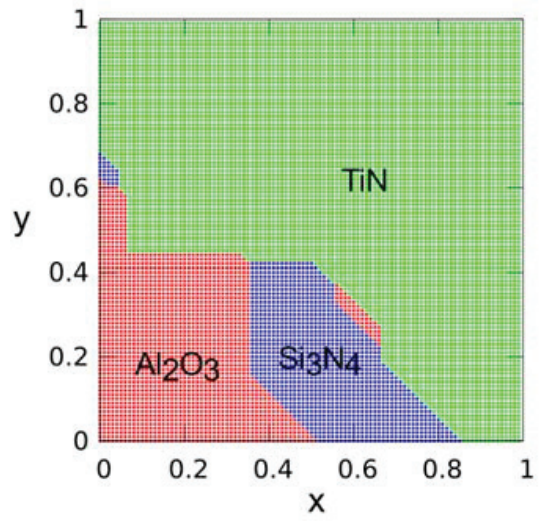

FIGURE 3. Crystalline structure in the cross section $x=0.5$

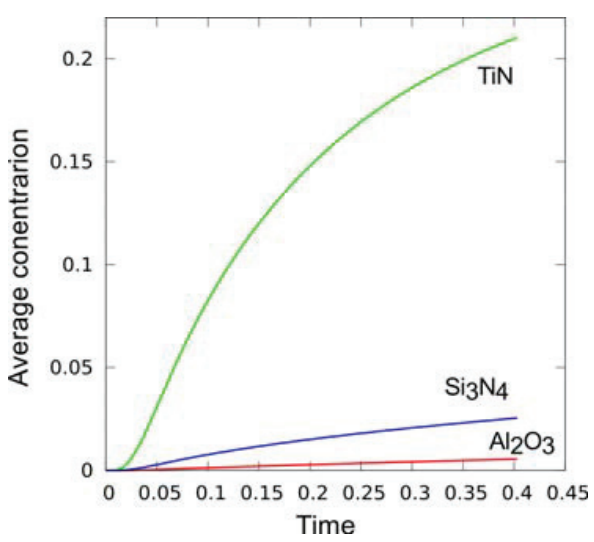

FIGURE 4. Average products concentration in the considerable domain

Firstly, the nitrogen excess, secondly, the reaction order. Average concentrations of the products demonstrate the same behavior (Fig. 4). Small amount of products is a result of early completion of calculations, because the compounds have occupied all available automata, but some of reactions were still in process. Another reason is the absence of diffusion. In our model all automata are isolated systems that do not exchange the matter with each other. Probably, the diffusion should be taken into account even for high rate processes. The third reason is temperature dependence. Real systems have strong temperature dependence, especially for the rates of chemical reactions. Therefore, this is the character of spatial temperature distribution that can have the significant impact on the formation of microcrystalline structure.

\section{CONCLUSIONS}

We have formulated the model of forming microcrystalline structure of coating deposited by magnetron sputtering. The model is based on the method of cellular automata. The results for a particular system were obtained. The modeling demonstrated that the initial conditions and the reaction order have a significant influence on the structure formation.

The work was supported by Fundamental Investigations Program of SB RAS for 2013-2016 (Project III.23.2.1).

\section{REFERENCES}

1. A. D. Korotaev and D. P. Borisov, Phys. Mesomech. 16(1), 73 (2013).

2. E. A. Holm and C. C. Battaile, JOM 53(9), (2001) 20.

3. I. Steinbach, Model. Simul. Mater. Sci. Eng. 17, 7 (2009).

4. M. H. Karapetyanc, Chemical Thermodynamics (Moscow, 1975).

5. M. Mikolaychuk and A. Knyazeva, Izv. Vuzov. Fizika 12(2), 89 (2009). 